\title{
FREQUENCY AND DIFFUSION MAPS FOR THE SNS RING*
}

\author{
Y. Papaphilippou ${ }^{\dagger}$ \\ Brookhaven National Laboratory, UPTON NY 11973, USA
}

\begin{abstract}
Using a single particle dynamics approach, the major magnetic non-linearities of the SNS accumulator ring are studied. Frequency maps are employed in order to display the global dynamics of the beam, for several working points. By means of diffusion maps the major resonances are explored and their bandwidth is estimated. The global tune diffusion coefficient is finally used to compare and choose the best working point.
\end{abstract}

\section{THE SNS ACCUMULATOR RING}

The SNS ring is designed to accumulate a high-intensity beam with a beam power of up to $2 \mathrm{MW}$. The ring lattice has a 4-fold symmetry and consists of FODO arcs and quadrupole doublet straight sections [1]. The beam dynamics is mostly dominated by multi-particle effects such as space-charge [2] and other collective instabilities (e.g. electron-proton [3]), which are mostly intensity dependent. On the other hand, the strict fractional beam loss, dictated by the permissible radiation level for hands-on maintenance [4], imposes a very good control and correction of all non-linear effects. This includes the ones coming from magnet imperfections, the impact of which is amplified due to the large beam emittance. Indeed, in the early commissioning stages where the beam intensity will be low, the dominant effects will be of a single-particle nature. One of them is the chromaticity: in order to reduce the effect of collective instabilities the longitudinal phase space is painted to a large momentum spread of $\delta p / p=0.7 \%$, with the bucket size set at $\delta p / p=1 \%$ and the momentum aperture at $\delta p / p=2 \%$. Taken the bucket size as the reference, the chromatic tune-shift is around \pm 0.08 , which is close to the space charge tune-shift of $0.15-0.20$. Another key issue for the performance of the SNS ring is the proper working point (w.p.) choice. After an extensive tuning campaign [5], we have concentrated on 4 potential w.p. candidates, due to optical matching and aperture considerations. Two of them have a tune-split of more than half a unit, in order to avoid strong coupling from spacecharge and/or magnet errors: $\left(Q_{x}, Q_{y}\right)=(6.3,5.8)$, the old "nominal" w.p. for which most of the past studies have been performed, and $\left(Q_{x}, Q_{y}\right)=(6.23,5.24)$. The other two are in a quadrant of the tune space where structural resonances are not present: $\left(Q_{x}, Q_{y}\right)=(6.23,6.20)$, close to the coupling resonance which avoids all the main 3rd order resonances and $\left(Q_{x}, Q_{y}\right)=(6.4,6.3)$. In order to choose the best w.p., with respect to the non-linear particle

\footnotetext{
${ }^{*}$ Work performed under the auspices of the US Department of Energy

† papap@bnl.gov
}

behavior, we use Laskar's frequency map analysis [6]. In brief, the frequency map is a detailed tune footprint. Due to the very high precision of the tune determination through a refined Fourier analysis using the NAFF algorithm, all the excited resonances appear as line distortions of the footprint. The tune variation along the particle trajectory can give a good estimation of the diffusion rate, which can then be mapped to the real space, for each particle initial condition. Finally, the average tune diffusion coefficient can be used as a global quality factor for comparison between the dynamics of the different designs.

\section{WORKING POINT COMPARISON}

We choose a standard SNS ring lattice matched for the four w.p. in study. The fringe fields in the ends of the quadrupoles are modeled as thin kicks using 5th order maps issued by MaryLie 5 [7]. Systematic and random magnet errors at the required level of $10^{-4}$ of the main magnet field are also included [8]. The chromaticity sextupoles are switched off in order to study the effect of the chromatic tune-shift and then tuned to give 0 chromaticity. In all the simulations, the RF voltage is set to 0 , for simplicity. Finally, space-charge forces are not included, as we would like at a first stage to study the single particle resonance effects to the SNS beam. A total of 1500 particles are uniformly distributed on the phase-space with zero initial momentum and different initial transverse coordinates, up to $480 \pi \mathrm{mm} \mathrm{mrad}$ (the transverse physical aperture) and tracked with the FTPOT module of the UAL environment [9]. They are given 9 different $\delta p / p$ values, from $+2 \%$ to $-2 \%$ corresponding to the beam momentum aperture. The tracking is conducted for 500 turns to provide a good precision in the tune determination.

Frequency maps for the w.p. $(6.4,6.3)$ and natural chromaticity are displayed in Fig. 1, where different colors correspond to different $\delta p / p$. The triangular shape of the maps reflects the octupole-like nature of the leading order quadrupole fringe fields [10], which apparently dominate the single-particle non-linear dynamics. The first evident observation is that the beam is crossing a multitude of excited resonant lines, which appear as distortions of the frequency map, due to the huge chromatic tune-shift of more than 0.3 , for $\pm 2 \%$. This can be clearly viewed in Fig. 2, where we zoom on one of the frequency maps of Fig. 1. For $d p / p=-1 \%$, the chromatic tune-shift moves the beam very close to the half-integer resonance which is excited by quadrupole errors and quadrupole fringe fields, as well. Its width is quite big and can be measured on the frequency map to be around 0.004. In addition, the structural 5th or- 


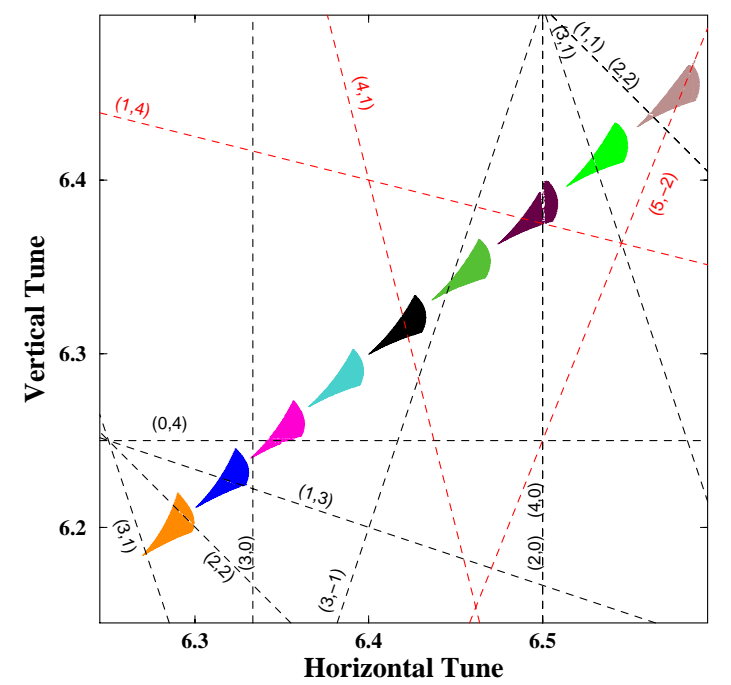

Figure 1: Frequency maps for the working point $(6.4,6.3)$, for 9 different $\delta p / p$, from $+2 \%$ (left bottom corner) to $-2 \%$ (right upper corner)

der resonance $Q_{x}+4 Q_{y}=32$ is excited. This effect can be mainly attributed to first order off-momentum "feeddowns" from the dominant dodecapole error in the edges of the quadrupoles and a small contribution from the systematic decapole error on the dipoles. A complementary feature of the frequency map is the diffusion map (Fig.2). One may compute the tune difference along a trajectory for two consecutive time-spans. The difference of the two values is a good indication of the particle diffusion. Then, a plot of the real space can be produced, mapping each initial condition with the diffusion coefficient, using a different color code. In this particular case, the grey dots correspond to stable particles, the green to weakly unstable and the blue/purple/brown to very unstable, most of them excited due to the half-integer resonance (outer stripe of initial conditions) and a few of them due to the 5th order normal decapole resonance. The frequency - diffusion maps for the w.p. $(6.23,5.24)$ and on-momentum particles are given in Fig. 3. In this case, the phase space is quite perturbed, as the w.p. is very close to the crossing of major 4th order resonances which are mostly excited by the quadrupole fringe fields. This can be also seen in the diffusion map, where the unstable area occupies more than half of the initial conditions space.

Finally, the frequency - diffusion map for the w.p. $(6.3,5.8)$ and $\delta p / p=1 \%$ is shown in Fig. 4. This map puts in evidence the major limitation of this w.p., i.e. the excitation of the structural coupling resonance $(1,1)$, due to quadrupole fringe fields and/or skew quadrupole errors. This resonance in combination with the space-charge can enhance particle loss to a level as high as $10 \%$ [2].

In order to compare the performance of each w.p., we use the tune diffusion indicator, which is computed by the average of the tune differences used for the construction of the diffusion maps and normalized by the initial emittances, for all the integrated orbits [6]. This indicator is correlated
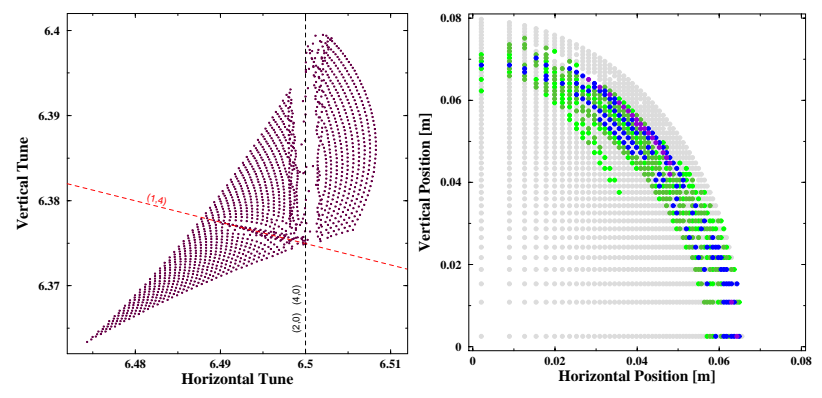

Figure 2: Frequency (left) and diffusion map (right), for the working point $(6.4,6.3)$ and $\delta p / p=-1 \%$. The different colors on the diffusion map represent different tune differences in logarithmic scale.
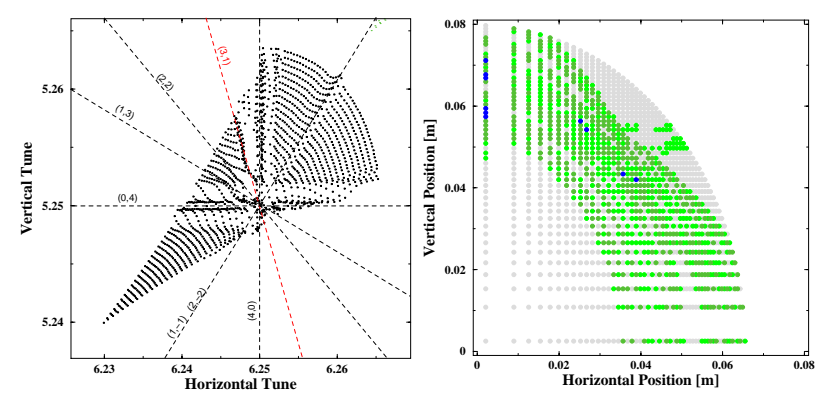

Figure 3: Frequency (left) and diffusion map (right) for the working point $(6.23,5.24)$ and $\delta p / p=0$.

with other global chaos indicators, as the resonances driving terms norm and the dynamic aperture [11]. We plot the value of the tune diffusion coefficient versus the $\delta p / p$ for all w.p., on the top of Fig. 5. The pick values on the diffusion indicators, for all w.p., correspond to areas of the phase space that are perturbed due to 4 th order resonances, showing once more the destructive effect of quadrupole fringe fields. The doted lines on the plots represent the average values of the diffusion indicators for all tracked momentum spreads. It is clear that $(6.23,6.20)$ is the best w.p. choice, followed by $(6.4,6.3)$. Their performance can be further improved by using the available multi-pole correctors [12], for correcting the normal and skew 3rd order resonances, in the case of $(6.4,6.3)$, and the 4 th order normal resonances
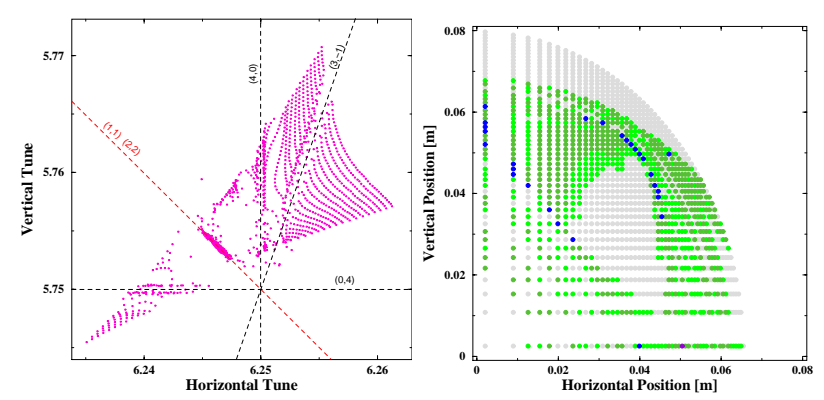

Figure 4: Frequency (left) and diffusion map (right), for the working point $(6.3,5.8)$ and $\delta p / p=1 \%$. 

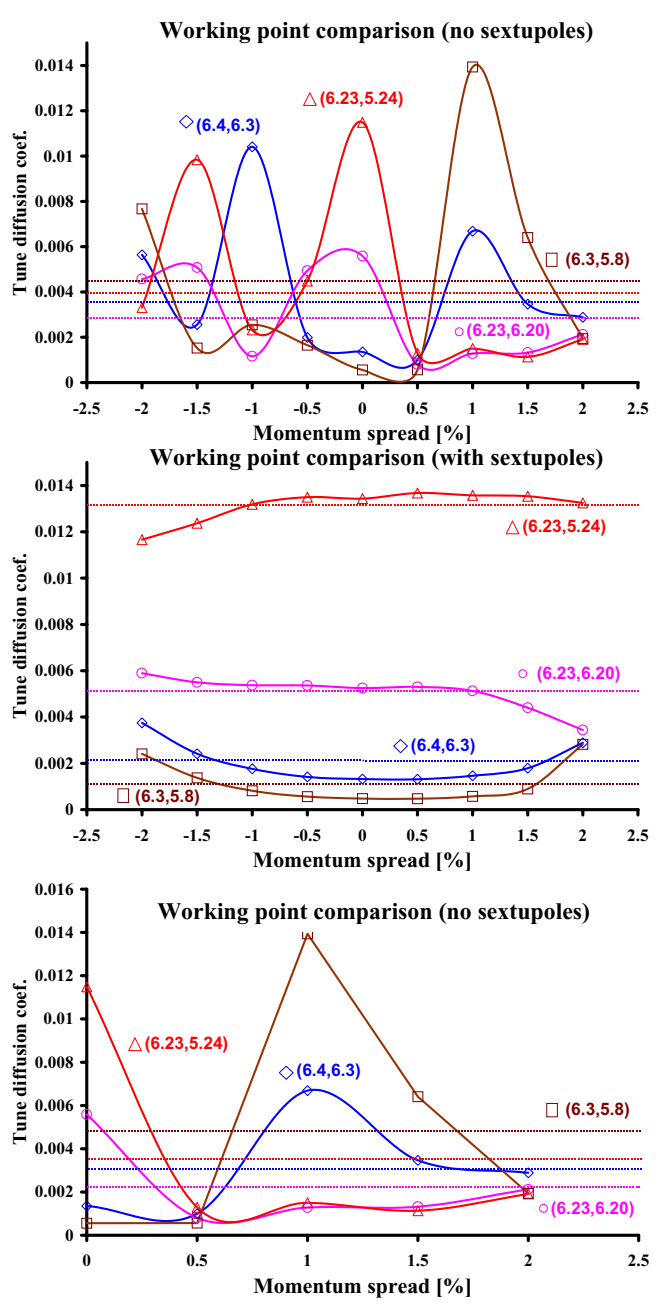

Figure 5: Tune diffusion coefficients for all working points versus the different momentum spreads, for natural chromaticity (top), for zero chromaticity (center) and for natural chromaticity and only positive momentum spread (bottom). The dashed lines are the average of the coefficient over all momentum spreads.

in the case of $(6.23,6.20)$. The other two w.p. have the disadvantage of crossing major structural coupling and 4th order resonances, which are very difficult to correct.

In the center of Fig. 5, we plot the tune diffusion coefficient when using the 4 families of chromaticity sextupoles [13] in order to set the chromaticity to 0 . In that case, the chromatic tune-shift is completely canceled and all the particles with different $\delta p / p$ are located in the same area of the tune diagram. This is reflected in the very weak dependence of the tune diffusion coefficient with respect to the momentum spread. Furthermore, the values of the average diffusion coefficient are almost equal to the ones for $\delta p / p=0$, when the chromatic sextupoles are switched-off. This proves the very small non-linear effect introduced by the chromaticity sextupoles. Finally, note that, in this case, $(6.3,5.8)$ seems to be the best w.p. choice. Nevertheless, this is not a fair comparison, as the space-charge force depresses the tunes. A better picture of the w.p. performance can be estimated by the average tune diffusion coefficient of all particles with positive momentum spread and nonzero chromaticity (at the bottom of Fig. 5). The results are pretty much the same as in the case of natural chromaticity.

\section{CONCLUSION}

The best performance with respect to the SNS ring single particle dynamics is expected from the w.p. $(6.23,6.20)$. This is also confirmed by space-charge simulations [2]. A question may arise regarding the effect of the coupling Montague resonance $2 Q_{x}-2 Q_{y}=0$ : indeed, for certain type of beam distributions (as the square shaped beam produced by anti-correlated painting [14]), this resonance can be a problem. On the other hand, for a round beam (for correlated painting [14]), this resonance is not excited. A possible second choice for avoiding coupling would be a w.p. with integer tune-split, such as $(6.19,5.20)$, which avoids the destructive effect of 4 th order resonances observed for the w.p. $(6.23,5.24)$ (see also Fig. 3). The second question with respect to the choice of $(6.23,6.20)$ as the SNS ring w.p., comes from the possibility of crossing the integer resonances $\left(Q_{x}, Q_{y}=6\right)$. Taking into account that beam loss is enhanced when crossing the resonance in the coherent sense (see [2] for details), the succesfull 2MW beam operation with this w.p. seams feasible. A possible second choice when pushing for higher intensity would be the w.p. $(6.4,6.3)$, provided that the correction schemes will enable the correction of the $3 \mathrm{rd}$ and 4 th order integer resonances.

\section{ACKNOWLEDGMENTS}

I thank the BNL-SNS accelerator physics group for discussions and in particular D.T. Abell, N. Catalan-Lasheras, A. Fedotov and J. Wei. I would like to express my gratitude to J. Laskar for introducing me to frequency map analysis.

\section{REFERENCES}

[1] J. Wei, et al., PRST-AB, 3:080101, 2000; J.Wei et al., these proceedings; W.T. Weng, et al., these Proceedings.

[2] A. Fedotov, et al., these Proceedings.

[3] M. Blaskiewicz, EPAC 2000, Vienna, June 2000;

[4] N. Catalan-Lasheras, these Proceedings.

[5] J. Holmes, et al., these Proceedings.

[6] J. Laskar, Astron. Astroph. 198, 341, 1988; Icarus 88, 266, 1990; Physica D 67, 257 1993; NATO-ASI NATO-ASI, S'Agaro, Spain, 1996; J. Laskar, et al., Physica D 56, 253, 1992; H.S. Dumas and J. Laskar, Phys. Rev. Let. 70, 2975, 1993; Y. Papaphilippou, PAC'99, New York, 1999; D. Robin et al., Phys. Rev. Let. 85, 558, 2000; A.L. Robinson, CERN Courier, Vol 41, N.1, 2001.

[7] A. Dragt et al., MaryLie 5, Un. of Maryland, 2000.

[8] J. Jackson et al, BNL-AM-MD-303, 2001.

[9] N. Malitsky and R. Talman, AIP 391, 1996.

[10] Y. Papaphilippou and D.T. Abell, EPAC2000, Vienna, 2000.

[11] Y. Papaphilippou, EPAC 2000, Vienna, 2000.

[12] Y. Papaphilippou et al., this Proceedings.

[13] Tsoupas et al., EPAC 2000, Vienna, 2000.

[14] J. Beebe-Wang, et al., EPAC 2000, Vienna, 2000. 